\title{
Aspectos cinemáticos del mecanismo de mordida premaxilar en los géneros Cauque, Basilichthys y Austromenidia (Teleostei, Atherinidae)*
}

\author{
EDUARDO DE LA HOZ U. \\ Laboratorio de Zoología, Instituto de Biología \\ Universidad Católica de Valparaíso \\ Casilla 4059, Valparaíso, Chile
}

\begin{abstract}
RESUMEN. Se analiza el mecanismo de mordida premaxilar de distintas especies de los géneros Cauque, Austromenidia y Basilichthys, a través de modelos cinemáticos de cuatro barras, que permiten describir las características de los movimientos de las piezas bucales.

En Cauque y Austromenidia las trayectorias angulares de premaxilar y mandíbula son semejantes y muestran un incremento en la velocidad del premaxilar a medida que se aproxima a la posición de cierre, mientras que en las especies de Basilichthys el premaxilar se mueve muy poco en relación al desplazamiento de la mandíbula y mantiene una velocidad angular constante durante toda la trayectoria.

Se relacionan las características funcionales del mecanismo con las siguientes proporciones estructurales medidas en especímenes de varias especies: borde mandibular / distancia cuadrado-palatino, borde mandibular / longitud premaxilar, distancia cuadrado-palatino / protracción premaxilar máxima, y distancia cuadrado-palatino / borde mandibular.
\end{abstract}

Palabras clave: Atherinidae, morfología funcional, mordida premaxilar, alimentación.

\section{Kinematic ofthe premaxillary bite ofthe genera Cauque, Basilichthys and Austromenidia (Teleostei, Atherinidae)}

\begin{abstract}
The premaxillary bite mechanism of various species ofthe genera Cauque, Austromenidia and Basilichthys are analyzed through the four-bar linkage models; these models allow the description ofthe nature ofthe buccal structures movements.

In Cauque and Austromenidia the angular trajectories of premaxilla and maxilla are similar, they show an increasing premaxilla velocity towards the cIosing position. In the Basilichthys species, the premaxilla movement is shorter than that of the mandible, and its velocity is maintained constant during all the trajectory.

The functional characteristics of the mechanism are related to the following structural ratios: mandibular edge / quadrate-palatine distance, mandibular edge / premaxilla length, quadrate-palatine distance / maximal premaxillary protrusion, and quadrate-palatine distance / mandibular edge.
\end{abstract}

Key words: Atherinidae, functional morphology, premaxillary bite, feeding.

* Trabajo financiado por Proyecto FONDECYT N $721 / 92$. 


\section{INTRODUCCION}

Las características de los movimientos de una estructura están determinados, por un lado, por la configuración estructural del sistema y por otro, por la calidad funcional del sistema muscular.

Cuando se trata de movimientos que son transmitidos mediante piezas intermedias a elementos esqueléticos situados a cierta distancia, el diseño estructural de éstas adquiere especial relevancia, ya que de ellas depende directamente la transformación del movimiento del músculo, en principio lineal, en movimientos con trayectorias, magnitudes y velocidades que pueden ser muy diferentes.

El uso de modelos mecánicos en estudios de morfología funcional en peces ha permitido lograr la comprensión entre la configuración geométrica y la función de los sistemas esquelético-musculares (Aertz y Verraes, 1984; Aertz et al., 1987; Alexander, 1966,1967; Anker, 1974; Elshoud-Oldenhave, 1979; Lauder y Liem, 1981; Muller, 1987; Westneat y Wainwright, 1989; Westneat, 1990). Entre ellos, la utilización de modelos basados en sistemas mecánicos de cuatro barras se han demostrado particularmente útiles y predictivos en mecanismos de alimentación (Westneat, 1990).

La configuración del sistema esquelético-muscular de la región bucal en especies sudamericanas de Atherinidae, permite no sólo una variada magnitud de protracción premaxilar, sino también la posibilidad de realizar una función de morder accionando con el premaxilar. Esta capacidad de mordida premaxilar está basada en la transmisión del movimiento del maxilar, producido por acción del fascículo $\mathrm{Al}$ del músculo aductor mandibular, mediante la conexión ligamentosa entre maxilar y premaxilar, y por la movilidad de este último respecto del neurocráneo y respecto de la mandíbula. (De la Hoz y Vial, 1988, 1994).

El objetivo de este estudio es elaborar un modelo cinemático del mecanismo de mordida premaxilar que permita analizar las características funcionales del mecanismo y sus dependencias de los aspectos estructurales de los elementos esqueléticos involucrados, en diferentes géneros y especies de Atherinopsinae chilenos.

\section{MATERIALES Y METODOS}

Se utilizaron ejemplares de las siguientes especies y localidades:

- Basilichthys semotilus (Cope, 1874), 11 especímenes, con longitudes estándar (LS) entre 77.4 y 110.3 mm, colectados en el Río Loa, II Región.

- Basilichthys australis (Eigenmann, 1927), 12 ejemplares, con 98.2 a $114.2 \mathrm{~mm}$ de LS, provenientes del Río Bío-Bío, VII Región.

- Basilichthys microlepidotus (Jenyns, 1842), 11 ejemplares, con LS entre 75.1 y $142.2 \mathrm{~mm}$, del estero Limache, V Región.

- Cauque brevianalis (Günther, 1880), 15 especímenes, con LS entre 73.5 y $110.8 \mathrm{~mm}$, del estero Quintero, V Región.

- Austromenidia regia (Humboldt, 1821), 11 ejemplares, 92.0 a $139.1 \mathrm{~mm}$ de LS, colectados en Valparaíso, V Región.

Los especímenes fueron fijados y conservados en formol al $10 \%$ y tratados para tinción de cartílagos y huesos según Dingerkus y Uhler (1977), para descripción y mediciones de las estructuras bucales.

Las observaciones y mediciones se realizaron con ayuda de un microscopio estereoscópico Wild M-7, con un pie de metro con precisión de $0.02 \mathrm{~mm}$.

\section{RESULTADOS Y DISCUSION}

La acción de mordida con el premaxilar es llevada a cabo mediante el movimiento relativo del premaxilar respecto de la mandíbula. El premaxilar puede rotar, en el plano sagital, alrededor de la articulación ligamentosa que relaciona un punto del borde del extremo póstero-ventral del premaxilar con un punto del extremo póstero-dorsal del dentario (De la $\mathrm{Hoz}$ y Vial, 1988) (Fig. 1).

Para que este mecanismo sea ejecutado, es condición indispensable que el extremo anterior del premaxilar esté en algún grado de protracción, es decir, esté en una posición adelantada respecto del neurocráneo.

Como la mordida premaxilar puede realizarse a partir de cualquier estado de protracción, se ha elegido la posición de máxima protracción como punto de partida del mecanismo con el fin de permitir la comparación entre las especies. 

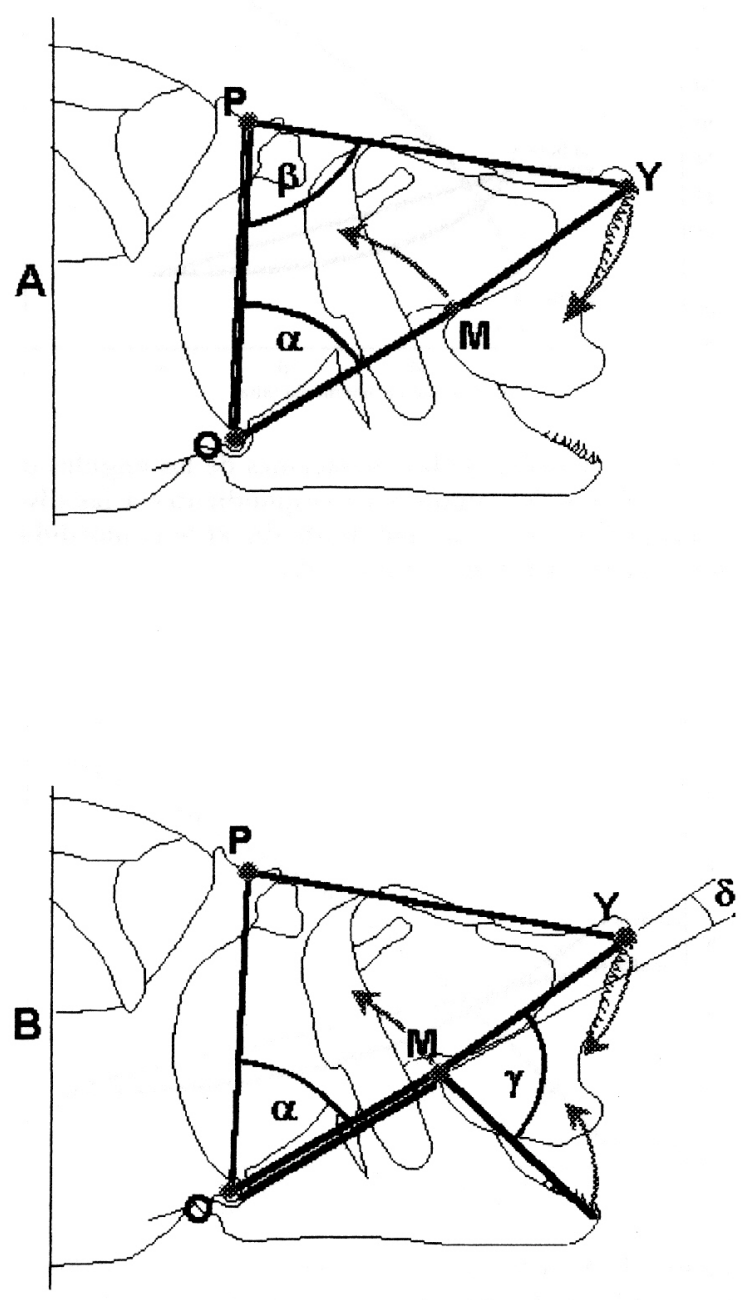

Figura 1. Esquemas de las estructuras bucales, indicando la configuración de los sistemas de cuatro barras utilizados. A: Posición del sistema con el segmento cuadrado-palatino (OP) como base inmóvil. B: Posición del sistema con el segmento del borde mandibular (OM) como base fija. PY: longitud del sistema de ligamentos rostrales en máxima protracción premaxilar; MY: segmento que representa la longitud del premaxilar; a: ángulo de posición mandibular respecto del eje cuadrado-palatino del suspensorio; 8: ángulo de posición del premaxilar respecto del eje cuadrado-palatino; r: ángulo de apertura bucal; 15: ángulo entre el eje premaxilar y el borde posterior de la mandíbula.
Para determinar las características funcionales de esta capacidad de mordida premaxilar, así como sus dependencias del diseño estructural de los elementos esqueléticos, se ha formulado un modelo cinemático, basado en un sistema de cuatro barras, utilizando la ecuación de Freudenstein (Hunt, 1990) que describe las relaciones entre ángulos y brazos de un sistema.

Dos aspectos son considerados para el análisis de la cinemática del mecanismo:

El primero, es la relación entre el movimiento de cierre mandibular, expresado en la variación del ángulo a entre el borde dorsal posterior de la mandíbula y el eje cuadrado-palatino, y el movimiento del extremo anterior del premaxilar, rotando en torno del punto de inserción proximal del sistema ligamentoso rostral, manteniendo la máxima protracción (Fig. 1 A).

El segundo, considera la relación entre las variaciones del ángulo mandibular (a) y las correspondientes variaciones del ángulo de mordida entre premaxilary mandíbula $(\gamma)$ (Fig. $1 \mathrm{~B})$.

La ecuación que describe las relaciones angulares del sistema de barras, manteniendo el segmento OP como base (Fig. 1 A) es:

$$
\begin{aligned}
\mathrm{R} 1 \times \cos \beta-\mathrm{R} 2 \times \cos (180-\alpha)+\mathrm{R} 3+\cos (\beta-(180-\alpha))=0 \\
\text { donde } \mathrm{R} 1=\mathrm{p} / \mathrm{s} \\
\mathrm{R} 2=\mathrm{p} / \mathrm{r} \\
\mathrm{R} 3=\left(\mathrm{c}^{2}-\mathrm{p}^{2}-\mathrm{r}^{2}-\mathrm{s}^{2}\right) / 2 \mathrm{rs}
\end{aligned}
$$

siendo

p: la distancia cuadrado-palatino (OP)

r: la longitud del sistema rostral en máxima protracción (PY)

s: la longitud del borde posterior de la mandíbula

(OM)

c: la longitud del eje premaxilar (MY)

A través de esta ecuación es posible determinar la magnitud del ángulo $\beta$ (que describe la posición del premaxilar respecto del suspensorio) en función del ángulo a (que determina la posición de la mandíbula respecto del suspensorio), o viceversa.

Para determinar la relación entre el ángulo a (entre mandíbula y suspensorio) y el ángulo y (entre premaxilary mandíbula) del sistema de cuatro barras de la Figura $1 \mathrm{~B}$, donde el segmento fijo corresponde ahora a OM, la ecuación de Freudenstein queda como: 
$\mathrm{R} 1 \times \cos \alpha-\mathrm{R} 2 \times \cos (\delta)+\mathrm{R} 3+\cos (\alpha-\delta)=0$

siendo $\delta$ el ángulo entre el segmento MY y la proyección de OM, y debiéndose reemplazar los elementos R1, R2 y R3 por:

$$
\begin{aligned}
& \mathrm{R} 1= \mathrm{p} / \mathrm{s}=\mathrm{OM} / \mathrm{MY} \\
& \mathrm{R} 2=\mathrm{p} / \mathrm{r}=\mathrm{OM} / \mathrm{OP} \\
& \mathrm{R} 3=\left(\mathrm{c}^{2}-\mathrm{p}^{2}-\mathrm{r}^{2}-\mathrm{s}^{2}\right) / 2 \mathrm{rs}=\left(\mathrm{PY}^{2}-0 \mathrm{M}^{2}-\mathrm{OP}^{2}-\mathrm{MY}^{2}\right) / \\
&(2 \mathrm{xOPxMY})
\end{aligned}
$$

La variación del ángulo $(\gamma)$ entre premaxilar y mandibula, corresponde al valor de $\gamma$ inicial en posición de máxima protracción con boca abierta, menos la variación del ángulo $\delta$.

La determinación de estas relaciones angulares en algunos especímenes de especies pertenecientes a los distintos géneros, se realizó ingresando los datos correspondientes a mediciones reales de las piezas esqueléticas y simulando la variación del ángulo mandibular $\alpha$ a partir de la condición de máxima apertura bucal.

En las Figuras 2 a 5, se grafican las variaciones de los ángulos $\alpha$ y $\beta$, junto a los valores obtenidos del ángulo maxilo-premaxilar $(\gamma)$, para cada valor de ex. durante la realización del mecanismo de mordida.

Tanto en Cauque (Fig. 2) como en Austromenidia (Fig. 3), las líneas que representan las variaciones de los ángulos $\alpha$ y $\beta$, se cruzan en un punto, mientras que en las especies de Basilichthys (Figs. 4 y 5) ambas líneas permanecen separadas. Esto significa que en el caso de Cauque, la posición de mordida premaxilar (cuando $\gamma=0$ ) se consigue con igual desplazamiento angular de la mandíbula y del premaxilar; y en Austromenidia, incluso el desplazamiento angular del premaxilar es ligeramente superior al de la mandíbula.

En las especies de Basilichthys, en cambio, esta posición se obtiene con un desplazamiento del extremo del premaxilar bastante menor que el mandibular.

Estas diferencias encontradas en las magnitudes relativas de desplazamiento de los premaxilares durante la mordida entre los distintos géneros, coinciden con lo descrito por De la Hoz y Vial (1994) a partir de un modelo cinemático distinto.

Otra diferencia importante se produce al compa rar la forma de las curvas del ángulo y y de la variación del ángulo $\alpha$. Al hacer variar el ángulo mandibular $\alpha$ de manera constante, tanto en Cauque

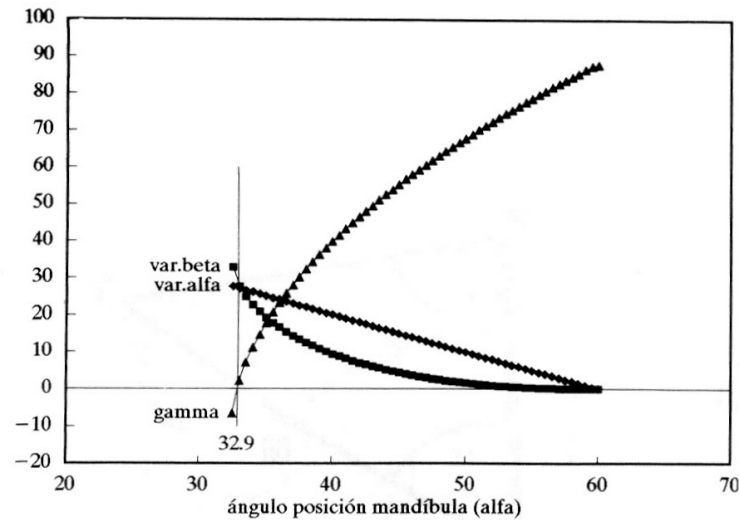

Figura 2. Gráfico de las variaciones de los ángulos $\alpha$ y $\beta$ y valores del ángulo y correspondientes a las distintas posiciones de la mandíbula durante la mordida premaxilar en Cauque brevianalis.

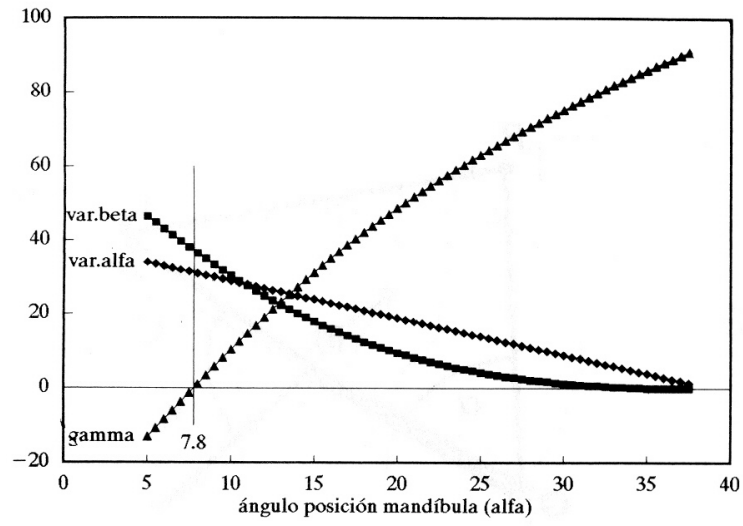

Figura 3. Gráfico de las variaciones de los ángulos $\alpha$ y $\beta$ y valores del ángulo y correspondientes a las distintas posiciones de la mandíbula durante la mordida premaxilar en Austromenidia regia.

como en Austromenidia, la variación de $\gamma$ no es lineal, sino que a medida que la posición de la mandíbula es más cerrada esta variación es mayor, lo que es especialmente notorio en Cauque. En cambio, en las especies de Basilichthys, la variación constante del ángulo mandibular $\alpha$, produce una variación de $\gamma$ prácticamente lineal (Figs. 4 y 5).

Tales comportamientos de las variaciones angulares significan que, tanto en Cauque como en Austromenidia, el movimiento de cierre del premaxilar se ve acelerado hacia la posición de mor- 


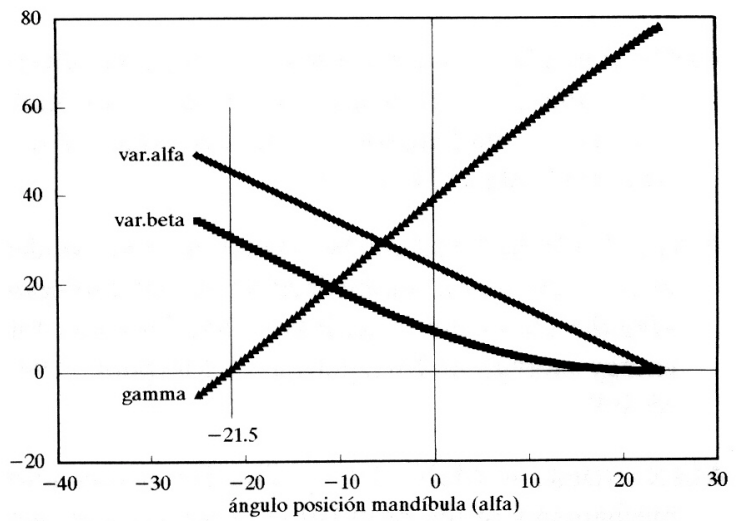

Figura 4. Gráfico de las variaciones de los ángulos $\alpha$ y $\boldsymbol{\beta}$ y valores del ángulo $\boldsymbol{\gamma}$ correspondientes a las distintas posiciones de la mandíbula durante la mordida premaxilar en Basilichthys microlepidotus.

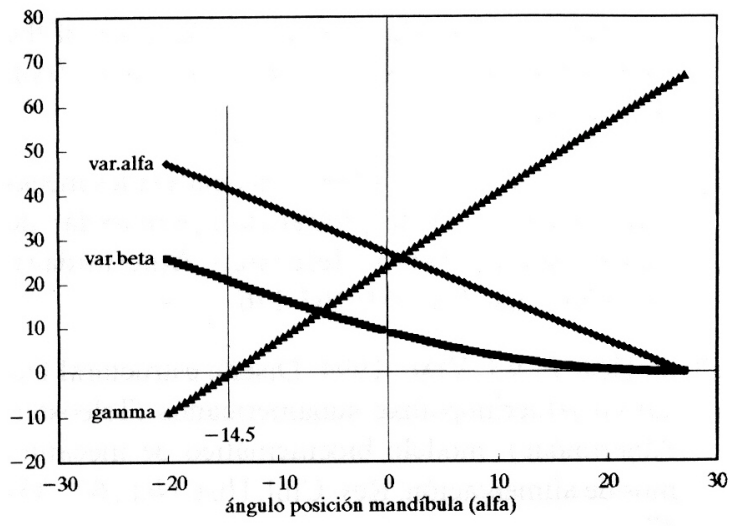

Figura 5. Gráfico de las variaciones de los ángulos $\alpha$ y $\boldsymbol{\beta}$ y valores del ángulo $\boldsymbol{\gamma}$ correspondientes a las distintas posiciones de la mandíbula durante la mordida premaxilar en Basilichthys australis.

dida, considerando que el movimiento de la mandíbula se realiza de manera constante. Esto puede interpretarse como una adaptación funcional de estos géneros, en orden de hacer más eficiente en cuanto a rapidez este mecanismo de captura de presas.

El análisis de las proporciones de las magnitudes estructurales que incluye la ecuación de Freudenstein (R1 y R2) muestra las diferencias entre los distintos géneros estudiados (Tabla 1).
Tabla 1. Valores promedio y (desviación estándar) de las proporciones: OP/OM, OP/OY, OM/OP, y OM/MY.

\begin{tabular}{lccccc}
\hline & $\mathrm{N}$ & $\mathrm{OP} / \mathrm{OM}$ & $\mathrm{OP} / \mathrm{OY}$ & $\mathrm{OM} / \mathrm{OP}$ & $\mathrm{OM} / \mathrm{MY}$ \\
\hline A. regia & 10 & $\begin{array}{c}1.450 \\
(0.156)\end{array}$ & $\begin{array}{c}0.807 \\
(0.104)\end{array}$ & $\begin{array}{c}1.015 \\
(0.057)\end{array}$ & $\begin{array}{c}0.697 \\
(0.066)\end{array}$ \\
\hline C. bre- & 15 & 1.382 & 0.834 & 0.744 & 0.728 \\
vianalis & & $(0.107)$ & $(0.055)$ & $(0.041)$ & $(0.054)$ \\
\hline B. & 14 & 2.184 & 0.501 & 1.514 & 0.459 \\
australis & & $(0.111)$ & $(0.027)$ & $(0.083)$ & $(0.023)$ \\
\hline $\begin{array}{l}\text { B. } \\
\text { semotilus }\end{array}$ & 11 & 2.220 & 0.511 & 1.677 & 0.458 \\
\hline B. micro- & 07 & $(0.220)$ & $(0.078)$ & $(0.136)$ & $(0.060)$ \\
lepidotus & & $(0.156)$ & $(0.067)$ & $(0.177)$ & $(0.033)$ \\
\hline
\end{tabular}

Al plotear Rl =OP/OM vs R2=OP/PY (Fig. 6), las especies de Basilichthys se distinguen claramente por los valores más altos de ambas proporciones, producto de la reducción proporcional de la magnitud de protracción premaxilar (PY) y de la longitud del borde dorsal posterior de la mandíbula (OM). $A$. regia y $C$. brevianalis poseen iguales proporciones de OP/OM, siendo levemente inferiores las magnitudes de OP/PY en esta última especie.

Al comparar las proporciones OM/MY y OM/ OP (Fig. 7) se observa una situación opuesta a la anterior. Las especies de Basilichthys poseen los valores más bajos de ambas proporciones, como consecuencia de magnitudes proporcionales más reducidas del borde mandibular (OM) y mayores longitudes del premaxilar (MY).

Los resultados de la aplicación de los modelos cinemáticos de cuatro barras permiten precisar la naturaleza de los movimientos realizados durante el mecanismo de mordida premaxilar. Además, la comparación de los sistemas estructurales en especies de distintos géneros de Atherinopsinae, permite apreciar distintas caracteristicas del funcionamiento del mecanismo, que pueden ser entendidas como modificaciones adaptativas dentro del repertorio de actividades de captura de presas. Cauque brevianalis es la especie que se presenta más especializada en rela- 


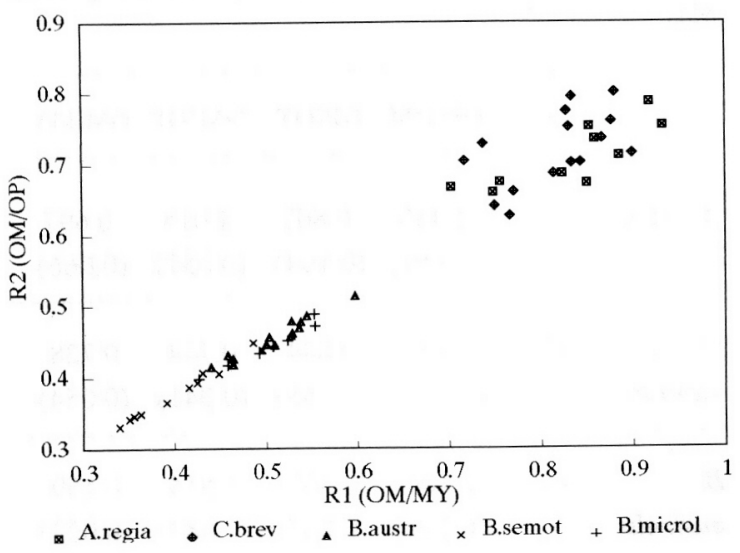

Figura 6. Representación gráfica de los valores de las proporciones OM/OP y OM/MY obtenidos de especímenes de todas las especies estudiadas.

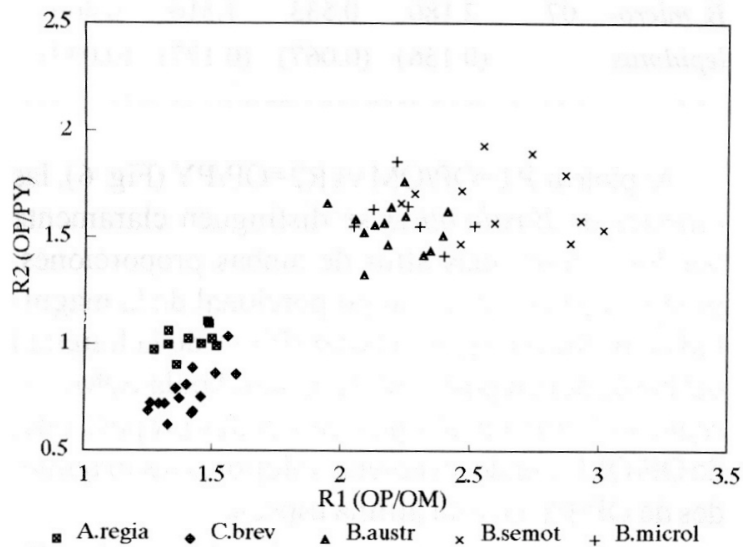

Figura 7. Representación gráfica de los valores de las proporciones OP/PY y OP/OM obtenidos de especímenes de todas las especies estudiadas.

ción a las características cinemáticas de la mordida premaxilar, con un mayor rango de posiciones de mordida y una mayor magnitud de desplazamiento y velocidad del premaxilar respecto de la mandíbula. Las especies del género Basilichthys poseen una posibilidad de mordida más restringida.

\section{REFERENCIAS BIBLIOGRAFICAS}

AERTZ, P. and W. VERRAES. 1984. Theoretical analysis of aplanar four bar linkage in the teleostean skull. The use of mathematics in biomechanics. Ann. Soc. Roy. Belg., 114: 273290.

AERTZ, P., J.W.M. OSSE and W. VERRAES. 1987. Model of jaw depression during feeding in Astatotilapia elegans (Teleostei: Cichlidae): Mechanisms for energy storage and triggering. J. Morphol., 194: 85-109.

ALEXANDER, R.McN. 1966. The functions and mechanisms of the protrusible upper jaws of two species of cyprinid fish. J. Zool. (London), 149: 288-296.

ALEXANDER, R.McN. 1967. The functions and mechanisms of the protrusible upper jaws of some acanthopterygian fish. J. Zool. (London), 151: 4364.

ANKER, G.Ch. 1974. Morphology and kinetics of the stickleback, Gasterosteus aculeatus. Trans. Zool. Soco (London), 32: 311-416.

DE LA HOZ, E. y J. VIAL. 1988. Potencialidades mecánicas del sistema de protracción premaxilar de Austromenidia regia (Teleostei- Atherinidae). Rev. Chil. Hist. Nat.,61: 163-176.

DE LA HOZ, E. y J. VIAL. 1994. Diseño estructural bucal en Atherinopsinae sudamericanos (Teleostei, Atherinidae): modelo biocinemático de mecanismos de alimentación. Rev. Chil. Hist. Nat., 67: 3547.

DINGERKUS, G. and L.D. UHLER. 1977. Enzyme clearing of alcian blue stained whole vertebrates for demonstration of cartilage. Stain Technol., 52: 229-232.

ELSHOUD-OLDENHAVE, M.J.W 1979. Prey capture in the pike perch Stizostedion lucioperca (Teleostei, Percidae): a structural and functional analysis. Zoomorphologie, 93: 1-32.

HUNT, K.H. 1990. Planar displacements through three and more locations. The four-bar linkage function generator. Algebraic synthesis. In «Kinematic geometry of mechanisms». 187-190. Oxford University Press, N.Y. 
LAUDER, G.V. and K.F. LIEM. 1981. Prey capture by Luciocephalus pulcher: implications for models of jaw protrusion in teleost fishes. Env. Biol. Fish., 6: 257-268.

MULLER, M. 1987. Optimization principIes applied to the mechanism of neurocranium levation and mouth bottom depression in bony fishes (Halecostorni). J. Theor. Bio., 126: 343-368.
WESTNEAT, M.W. and P.C. WAINWRIGHT. 1989. The feeding mechanism of Epibulus insidiator: evolution of a novel functional system. J. Morphol.,202: 129-150.

WESTNEAT, M.W. 1990. Feeding mechanics of teleost fishes (Labridae; Perciformes): a test of four-bar linkage models. J. Morphol., 205: 269295.

Recibido el 16 de marzo de 1994. Aceptado el 25 de octubre de 1994. 